Editorial

\title{
Fragility Fractures in the Elderly Osteoporotic Patient and the Efficacy of Fracture Liaison Service Programs
}

\author{
Tarik Wasfie ${ }^{1,}{ }^{*}$, Avery Jackson III ${ }^{2}$, Cara Marie Brock ${ }^{2}$, Stefanija Galovska ${ }^{2}$, Jennifer Hille ${ }^{1}$
}

1. Ascension Genesys Grand Blanc, One Genesys Parkway, Grand Blanc, MI, USA; E-Mails: Tarik.Wasfie@ascension.org; Jennifer.Sunderman@ascension.org

2. Michigan Neurosurgical Institute, Grand Blanc, USA; E-Mails: ajackson@michneurosurgical.com; cbrock@michneurosurgical.com; sgalovska@michneurosurgical.com

* Correspondence: Tarik Wasfie; E-Mail: Tarik.Wasfie@ascension.org

Special Issue: Osteoporosis in the Elderly

\section{OBM Geriatrics}

2019, volume 3, issue 4

doi:10.21926/obm.geriatr.1904085
Received: November 07, 2019

Accepted: November 11, 2019

Published: November 13, 2019

\section{Keywords}

Ostroporosis; elderly; fragility; fracture; FLS

Osteoporosis is a skeletal disorder characterized by low bone mass which decreases bone strength, causing the bone to become fragile and brittle and thus increasing the risk of fracture [1]. Osteoporosis has a serious impact in the elderly with associated fragility fracture (defined by the WHO as fracture caused by injury that is insufficient to fracture a normal bone). The best example, and the most common, is a fall from standing, or no identifiable trauma. It is still unrecognized and underappreciated, despite the two million osteoporotic fractures and half million admissions to the hospital occurring annually in the United States and have a cost that has come to exceed one billion dollars annually [2]. Women by far outnumber the men, and it is estimated to effect 200 million women worldwide [1]. In the United States, among females 55 years and older, the cost of hospitalization of osteoporotic fracture is greater than that of myocardial infarction, stroke and breast cancer combined [3], and with increasing longevity in the United States and the world the

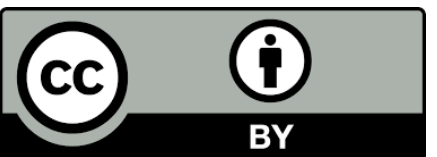

(C) 2019 by the author. This is an open access article distributed under the conditions of the Creative Commons by Attribution License, which permits unrestricted use, distribution, and reproduction in any medium or format, provided the original work is correctly cited. 
burden of the osteoporotic fragility fracture will only increase and require an immediate effective program to prevent, control, and reduce the upward trend. Fracture liaison service (FLS) programs are a clinical concept directed toward follow up of the osteoporotic patient at regular intervals with the aim of improving bone health and preventing fractures and refracture rates in those patients, ultimately improving outcomes. It was initially established in 1995 [4] and has gained popularity recently. In our community of 400 beds with a level 2 trauma service, we admitted an average of 850-900 trauma patients annually with a mean age of 62 years [4]. Our FLS program was established in 2015 by the neurosurgical section (Michigan Neurosurgical Institute) to care for patients with osteoporosis as well as osteoporotic patients with fragility fractures. It is run by a neurosurgeon champion and physician assistants to follow up with those patients at regular intervals to check and improve any metabolic deficiency as well as treat with hormonal and pharmacological agents when required. The program was detailed in prior publication $[4,5]$. The emphasis is on improving patient physical strength by regular exercise and reducing the fall rate by improving patients surrounding environment with aim to reduce fractures and refracture rates. In brief, it included multiple visits by the patients to the clinic at 3 to 6 -month intervals over a period of two years for clinical and laboratory evaluation, followed by treatment with supplemental calcium and vitamin $D$, with hormonal and/or pharmacological agents to improve bone activity to be added as needed. Additionally, dietary instruction; weight loss, physiotherapy and environmental improvement in the patient's living surroundings are implemented. When the program started in 2015, we saw 215 osteoporotic patients with vertebral compression fractures between 2015-2017. We collected the data on those patients and their refracture rate following initial vertebral fracture and compared to 150 patients with similar diagnosis seen before the program was established to evaluate the improvement in the outcome of refracture rates and in patients with vertebral compression fractures [6]. The result was encouraging as improvement in fracture rates for the FLS group was significantly lower (37\%) compared to refracture rate in the pre-FLS patients $(56 \%) \quad(p=0.01)$. This improvement was even more pronounced in the postmenopausal woman. These results reflect the benefit of the program as one of the effective options to improve the health of the elderly osteoporotic patient and reduce fracture and refracture rates. Currently the programs are geared toward inclusion of all patients with osteoporotic fragility fractures (vertebral, rib, long bone and pelvic fractures) and to establish itself as a prime source of prevention.

\section{Author Contributions}

T. Wasfie: Author/Investigator; A. Jackson: Champion of FLS Program; C. Brock \& S. Galovska: PA Contribution to FLS Program; J. Hille: Data Processing/Manuscript.

\section{Competing Interests}

The authors have declared that no competing interests exist.

\section{References}

1. Igbal MM. Osteoporosis epidemiology, diagnosis and treatment. South Med J. 2000; 93; 2-18. 
2. Burge R, Dawson-Hughes B, Solomon DH, Wong JB, King A, Tosteson A. Incidence and economic burden of osteoporosis related fracture in the US 2005-2008. J Bone Miner Res. 2007; 22; 465-475.

3. Singer A, Exuzides A, Spangler L, O'Malley C, Colby C, Johnston K, et al. Burden of illness for osteoporosis fractures compared with other serious disease among postmenopausal women in the United States. Mayo Clin Proc. 2015; 90; 53-62.

4. Wasfie T, Jackson A, Shapiro B. Fractured ribs in the elderly: Chapter in book Elderly Care; options, challenges and trends. Editor D. Nielson, Nova 2018; Chapter 6; 143-176.

5. Miller AN, Lake AF, Emory CL. Establishing a fracture liaison service: An orthopedic approach. J Bone Joint Surg Am. 2015; 97; 675-681.

6. Wasfie T, Jackson A, Brock C, Galovska S, McCullough JR, Burgess JA. Does a fracture liaison service program minimize recurrent fragility fractures in the elderly with osteoporotic vertebral compression fractures? Am J Surg. 2019; 217: 557-560.

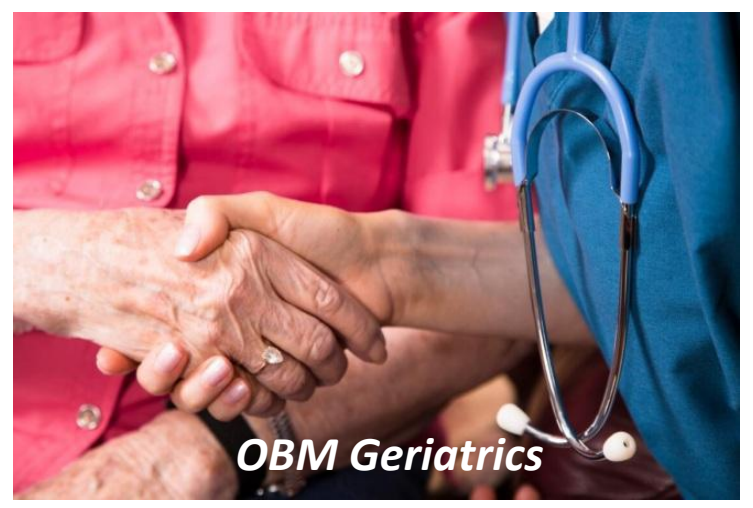

Enjoy OBM Geriatrics by:

1. Submitting a manuscript

2. Joining in volunteer reviewer bank

3. Joining Editorial Board

4. Guest editing a special issue

For more details, please visit: http://www.lidsen.com/journals/geriatrics 